\section{ECONOMICS}

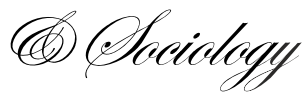

\author{
Claudia Hübner, \\ University of Greifswald, \\ Greifswald, Germany, \\ E-mail: claudia.buebner@uni- \\ greifswald.de
}

Steffen Flessa, University of Greifswald, Greifswald, Germany, E-mail: steffen.flessa@unigreifswald.de

Received: December, 2015 1st Revision: March, 2016 Accepted: June, 2016

DOI: $10.14254 / 2071-$ 789X.2016/9-3/10
Hübner, C., Flessa, S. (2016), Reimbursement for Hospital-acquired Infections with Multidrug Resistant Organisms in German DRG System, Economics and Sociology, Vol. 9, No 3, pp. 111-118. DOI: 10.14254/2071-789X.2016/9-3/10

\title{
REIMBURSEMENT FOR HOSPITAL- ACQUIRED INFECTIONS WITH MULTIDRUG RESISTANT ORGANISMS IN GERMAN DRG SYSTEM
}

\begin{abstract}
Hospital-acquired infections (HAIs), especially caused by multidrug resistant organisms (MDROs) are one of significant cost drivers for healthcare systems worldwide, associated, inter alia, with additional costs for hospitals.

An empirical study was conducted to analyse whether changes in German DRG system led to a better coverage of increased expenditure due to HAIs and the related prevention procedures.

784 patient records from six hospitals in Northeast Germany, documentation sheets as well as routine medical controlling data (including age, sex, length of stay, main diagnosis, secondary diagnoses, OPS-codes, DRG, effective cost weight) have been analysed.

Study population showed overall an increased length of stay but higher in the MDRO-positive group. Average cost weight was increased by 1.74 , average effective cost weight by 2.13. However, MDRO-patients gather lower revenue per day on average.

The study demonstrated that MDRO-parameters are not fully incorporated in the applied DRG-coding procedures and hygiene expenditures are still underfunded.
\end{abstract}

JEL Classification: I11, I18

Keywords: DRG-system, multiresistant pathogens, hospital acquired infections, reimbursement, infection control.

\section{Introduction}

Hospital-acquired infections (HAIs) are of immense clinical, epidemiological and ethical importance for healthcare systems worldwide (ECDC, 2012; Jarvis, 1996). Many of these infections are caused by multidrug resistant organisms (MDROs), of which the Methicillin Staphylococcus aureus (MRSA) is the most common one. High economic burden of HAIs can be referred to increased co-morbidity and mortality resulting in prolonged hospital lengths of stay as the main cost driver (Cosgrove et al., 2005; de Kraker et al., 2011; Wenzel, 1995; Zimlichman et al., 2013). Multidrug resistant pathogens complicate treatment procedures considerably and lead to significantly longer hospital lengths of stay (de Kraker et al., 2011). In 2008, the European Centre for Disease Prevention and Control (ECDC) estimated 171,200 nosocomial MRSA infections, 5,400 attributable excess deaths and more 
than 1 million excess days of hospitalization per year which goes along with 380 million Euros excess in-hospital costs per year (ECDC, 2012).

To combat the spread of multiresistant bacteria, hospitals are confronted with additional costs due to hygienic measures, e.g. admission screening, and precaution isolation of patients (Herr et al., 2003; Hubner et al., 2014; Wilcox, 2004). A few studies demonstrate a positive cost-benefit ratio of theses hygiene preventive measures (Farbman et al., 2013). Nevertheless, these additional costs are only partly accounted in hospital financing systems (Vegni et al., 2004). The financial impact of nosocomial infections on reimbursements under prospective Diagnosis Related Group (DRG) conditions is already long controversially debated (DeWitt, 1987). MDROs present a new challenge not least because of their growing clinical importance. However, there are hardly any scientific studies conducting the impact of MDRO-infections on the DRG-system.

\section{Encoding of multiresistant organism in the German DRG-system}

The German DRG System was introduced in 2003 and contains a diagnosed-regarded, lump-sum classification system. Each DRG corresponds with an economically-comparable case-group specified by the clinical diagnosis, diagnostic and therapeutic interventions as well as patients' comorbidities and subsequent complications. Key elements of a DRG are the mean length of stay with a lower and an upper limit, which indicates discount or surcharge rates, and the cost weight. There are ICD-10-based secondary diagnoses for the coding of comorbidities as well as OPS-codes for special performed procedures.

Over the past years several adjustments of the DRG system have been made, whereby also hospital acquired infections with multidrug-resistant pathogens should be better reflected. Originally, these costs were not included in DRGs and therefore not covered (Vegni et al., 2004). A positive MDRO-status can be indicated by the secondary diagnosis U80.0! or U81.0! in combination with Z22.3 Z29.0. (colonization) or B.95.6 (infection). For the isolation of MDRO-positive patients, the OPS 8-987! should be used if minimum requirements are met (documented additional expenses of at least 2 hours per day, e.g. for special experienced staff, screening, isolation and decolonization measures, over at least 7 days). Since 2007 specific complex DRG for MDRO-associated diseases exist for encoding.

The encoding of MDOR-relevant parameters may have an impact on the hospital revenue, as the following example shows (Table 1).

Table 1. Example of MDRO-adapted DRG-encoding

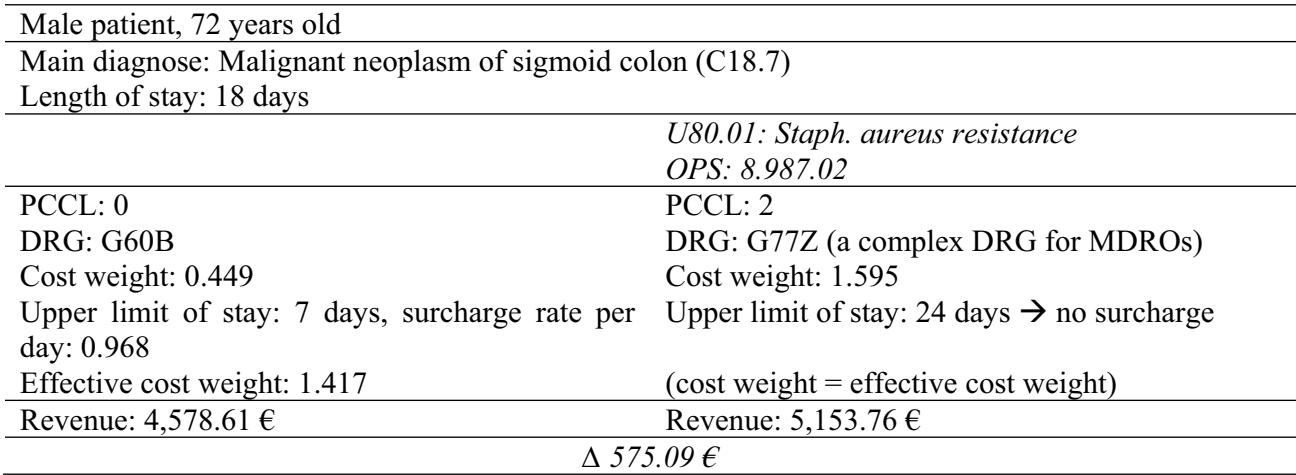


The example of Table 1 shows a difference of 575.09 Euro in favour of encoding of MDRO-parameters. This revenue growth is relatively low compared to the additional cost due to hygienic prevention measures and other therapeutic procedures (Herr et al., 2003; Hubner et al., 2014). Furthermore, encoding of MDRO-parameters does not always result in a revenue relevance, sometime it can be even revenue reductive (de Zeeuw and Baberg, 2009). If the required conditions for OPS 8-987! can not be achieved, no MDRO-relevant encoding is possible at all. Finally, carefully documented patient records are prerequisite (Chin et al., 2013).

\section{Study}

\subsection{Aim}

There are hardly any empirical analyses investigating whether the actual changes in the German DRG system regarding the encoding of MDRO-parameters have led to a better coverage of increased expenditure due to HAIs and its precaution measures. The study addresses this health-politically important issue by analysing the mechanism of coding of MDRO-cases in clinical routine and its effects on the clinical claim reimbursement. The analysis is based on real hospital data.

\subsection{Study design}

The evaluation was integrated in HARMONIC (Harmonized Approach to avert Multidrug-resistant Organisms and Nosocomial Infections), an 2-years investigation study (2012-2014) of the implementation of a standardized MDRO-hygienic management in six acute care hospitals in the "Health Region Baltic Sea Coast" in Mecklenburg-WesternPomerania in Northeast Germany. The detailed study protocol has been published elsewhere (Gerlich et al., 2015).

\subsection{Data}

As part of the economic evaluation 784 patient records consisting of data of several questionnaires and documentation sheets (including microbiology results, provided hygiene expenses, isolation periods, structural conditions of hospitals), which were developed specifically for the study, as well as routine data of the hospital medical controlling department (including age, sex, length of stay, main diagnosis, secondary diagnoses, OPScodes, DRG, effective cost weight) have been analysed. It included data of all in the study wards hospitalised risk patients, i.e. patients who have been screened for MDRO. For the two groups (MDRO-positive and MDRO-negative patients) an approximately equal severity of the major diagnosis can be expected, which allowed analysing the pure MDRO-effect.

\section{Results}

\subsection{Study population}

Of all 784 patients included in the analysis 56 were MDRO-positive (7.1\%) and 728 MDRO-negative (92.9\%). The distribution between the 6 hospitals was not uniform, so the MDRO-share ranged from $2.1 \%$ to $9.5 \%$. Together, 458 patients $(58.4 \%)$ were male and $322(41.1 \%)$ female, where in at the MDRO-positive group the proportion of men was 
significantly increased (71.1\%). The average age was 65.3 years in the MDRO-positive group and 63.7 years in the MDRO-negative group.

MDRO-cases were diagnosed in all ICD-10 disease classes (Table 2). However, there was an increased MDRO-status in patients with infectious and parasitic diseases as well as in patients with malignant neoplasm. Otherwise, in same diagnosis classes only a few MDROcases occurred.

Table 2. Distribution of ICD-10 diagnosis classes

\begin{tabular}{llcc}
\hline ICD-010 Diagnosis classes & $\begin{array}{c}\text { MDRO- } \\
\text { negative } \\
(\%)\end{array}$ & $\begin{array}{c}\text { MDRO- } \\
\text { positive } \\
(\%)\end{array}$ \\
\hline $\mathrm{A}+\mathrm{B}$ & Infectious and parasitic diseases & 3.0 & 10.7 \\
\hline $\mathrm{C}$ & Malignant neoplasm & 13.5 & 21.4 \\
\hline $\mathrm{D}$ & Diseases of the blood and blood-forming organs & 2.8 & 1.8 \\
\hline $\mathrm{E}$ & Endocrine, nutritional and metabolic diseases & 12.4 & 8.9 \\
\hline $\mathrm{F}$ & Mental and behavioral disorders & 1.2 & 1.8 \\
\hline $\mathrm{G}$ & Diseases of the nervous system & 5.4 & 0.0 \\
\hline $\mathrm{I}$ & Diseases of the circulatory system & 20.5 & 16.1 \\
\hline $\mathrm{J}$ & Diseases of the respiratory system & 4.0 & 5.4 \\
\hline $\mathrm{K}$ & Diseases of the digestive system & 12.1 & 14.3 \\
\hline $\mathrm{L}$ & Diseases of the skin and subcutaneous tissue & 3.2 & 7.1 \\
\hline $\mathrm{M}$ & Diseases of the musculoskeletal system and connective tissue & 3.3 & 3.6 \\
\hline $\mathrm{N}$ & Diseases of the genitourinary & 1.0 & 0.0 \\
\hline $\mathrm{R}$ & Symptoms and abnormal clinical and laboratory findings & 1.5 & 1.8 \\
\hline $\mathrm{S}+\mathrm{T}$ & $\begin{array}{l}\text { Injury, poisoning and certain other consequences of external } \\
\text { causes }\end{array}$ & 11.5 & 3.6 \\
\hline
\end{tabular}

\subsection{Length of stay in hospital}

Study population showed overall an increased length of stay (MDRO-positive group: 28.6 days and MDRO-negative group: 12.9 days) in comparison to mean length of stay in German hospital of 7.5 days (Statistika, 2014). MDRO-positive patients stayed in average 15.7 days longer in hospital, whereas their relative proportion varied greatly according the duration of stay (Figure 1). Most MDRO-negative cases (36.8\%) stayed in hospital less than 7 days, while in this short-stay-period only $21.4 \%$ of MDRO-positive cases were included. In contrary, $30.4 \%$ of MDRO-positive cases but only $8.8 \%$ of MDRO-negative cases had a very long length of stay of other 35 days. 


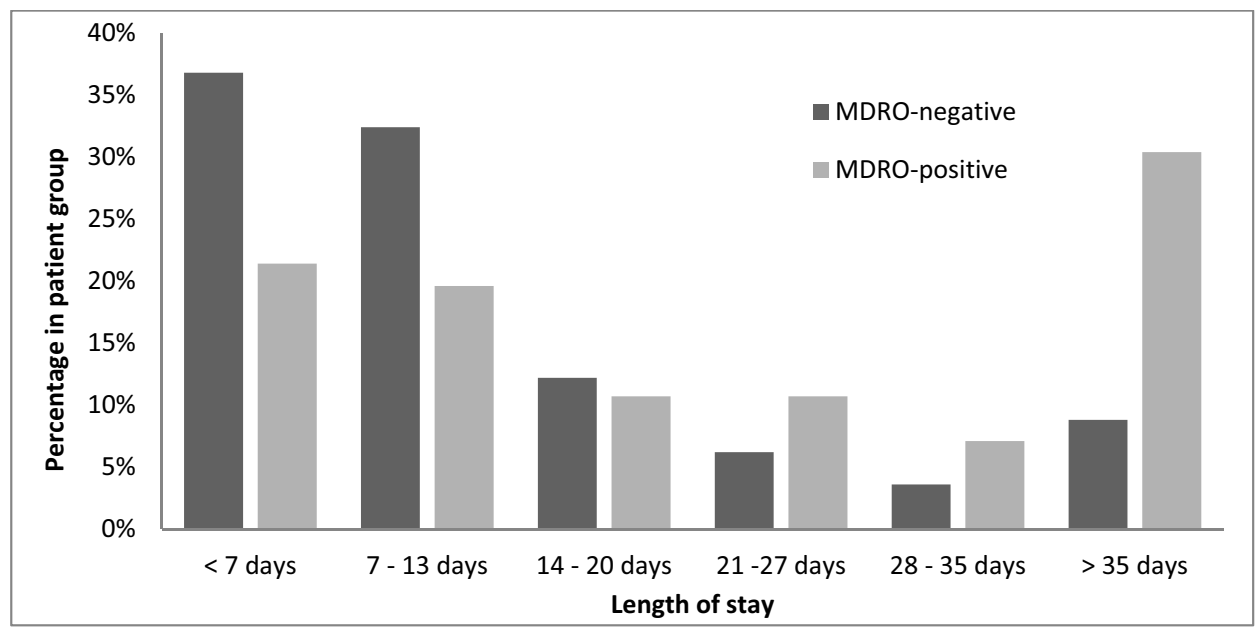

Figure 1. Distribution of patients with MDRO-status (negative or positive) in relation to length of stay

\subsection{Revenue data}

The analysis of the revenue data showed big differences between the MDRO-positive and MDRO-negative patient group (Table 3). Thus, in the MDRO-positive group the average cost weight was increased by 1.74 , the average effective cost weight by 2.13 . This resulted in different average revenue per case: 7,595.44 Euro (MDRO-negative) and 16,724.90 Euros (MDRO-positive). The calculated medians are always below the calculated values of the average. Nevertheless, the comparison between the two study groups shows increased values for the MDRO-positive group.

Regarding to the mean length of stay of 12.9 days of the MDRO-negative group, average revenue per day amounted in 623.40 Euros. Due to the longer mean length of stay (28.6 days) average revenues per day was lower in MDRO-positive group with 585.80 Euros. This difference results in a double-side problem, as for patients with a positive pathogen status additional costs of precaution measures occur (Hubner et al., 2014) that are offset by lower revenues per day (deficit in average: -37.60 Euros per day).

Table 3. Comparison of revenue data of MDRO-positive and MDRO-negative group

\begin{tabular}{|c|c|c|}
\hline & MDRO-negative $^{1}$ & MDRO-positive $^{1}$ \\
\hline Cost weight & $\begin{array}{c}2.30 \\
\text { (median: } 1.06 ; \\
\text { range: } 0.19-44.75 \text { ) }\end{array}$ & $\begin{array}{c}4.02 \\
\text { (median: } 2.06 ; \\
\text { range: } 0.50-25.73 \text { ) }\end{array}$ \\
\hline Effective cost weight & $\begin{array}{c}3.32 \\
\text { (median: } 1.50 \\
\text { range: } 0.21-45.74 \text { ) }\end{array}$ & $\begin{array}{c}7.08 \\
\text { (median: } 2.35 \\
\text { range: } 0.50-53.44 \text { ) }\end{array}$ \\
\hline Revenues per case & $\begin{array}{c}7,595.44 € \\
\text { (median: } 3,554.61 € ; \\
\text { range: } 590.32-139,511.21 € \text { ) }\end{array}$ & $\begin{array}{c}16,724.90 € \\
\text { (median: } 6,707.21 € ; \\
\text { range: } 1,503.91-161,386.48 €)\end{array}$ \\
\hline $\begin{array}{l}\text { Average revenues per day } \\
\text { (according to length of stay) }\end{array}$ & $623.40 €$ & $585.80 €$ \\
\hline
\end{tabular}




\section{Discussion}

The presented analysis focused on the impact on the clinical claim reimbursement of German hospitals by coding of MDRO-relevant parameters in the German DRG system. It could be shown that coding of MDRO-infections can lead to an increased reimbursement; however a cover of additional costs is not always granted. Thus, the study found similar conclusions as previous analyzes that were carried out before implementation of MDROrelevant changes in the DRG system (Resch et al., 2009; Wernitz et al., 2005). It must be concluded that the DRG system has not yet been optimally adapted to the coding of MDROinfections. The additional costs can not be completely transferred from the hospitals to the insurance providers. Therefore further changes might be necessary.

The coding of MDRO-positive cases leads to an increased average cost weight and average effective cost weight and consequently to increased average revenues per case compared to the MDRO-negative cases (Table 3). This effect is primarily due to the more complicated and therefore more costly events that occur more often in the MDRO-positive group. Considering the hygiene expenses as a disease severity independent component, the calculated average revenues per day confirm that no full reimbursement of costs is achieved.

The analysis showed a high percentage of incorrectly recorded MDRO-infections under DRG conditions. So, only $62.5 \%$ of all MDRO-cases have been coded with an U80.xx code indicating the MDRO-status. The OPS 8-987 was only coded in 41.1\% of MDRO-cases. For this purpose, various causes can be assumed.

First of all encoders' knowledge gaps must be taken into consideration. Here it greatly depends on whether the hospital has an own medical controlling department with full-time coding staff or the DRG-coding is carried out exclusively by the medical staff in addition to patient documentation. Chin et al. concluded recently that regular meetings between clinical and coding staff improve the quality and timeliness of medical documentation, ensure adequate communication with general practitioners and lead to appropriate funding (Chin et al., 2013). Updated coding guidelines need to be trained perennially. However, hospital doctors usually have a limited time budget, so they can not often participate in courses. The user-friendliness of the coding software also plays a role.

DRG-coding based on an extensive and time-consuming documentation of patient records. For example, in accordance for encoding of OPS 8-987 a 2-hour effort has to be proven. The documentation needs to be integrated in the process flow. The German SHI Head Association and the German Hospital Federation issued a special documentation sheet according OPS 8-987 which allows a rational and feasible documentation practice (DKG, 2007). Positively should be emphasized that the additional documentation time as part of a MDRO-measure is also recordable.

The applicability of encoding of the OPS 8-987 established a minimum requirement of an isolation period of at least 7 days. If a patient is less under isolation, the procedure key can not be recognized. This is one of the causes why in fewer than half of the analysed MDROcases the OPS 8-987 was not encoded. On the contrary, due to the hygiene regulations it can not be assumed that precaution measures have not been conducted; therefore the hospital has costs that are not reimbursed.

Another reason is that the encoding of the OPS 8-987 is not always associated with higher revenue. This concerns especially cases with a major diagnosis that already achieve an expensive DRG without MDRO-encoding. In particular, ventilated patients are affected by this. Switching to one of the MDRO-complex-DRGs would even reduce the reimbursement. Consequently, controllers adapt the encoding accordingly.

The objective of the analysis will determine which data should be evaluated. Due to business aspects a hospital manager will always focus on the revenue relevance in the DRG 
hospital billing. This reveals important limitations of infection control analyses by using DRG routine data. Therefore, routine controlling data may not be used solely for interpreting MDRO-status in hospitals. Moreover it requires other independent epidemiological surveillance documentations (Rosof, 2012). Similarly, costs of additional expenses due to MRDO measures can not determine alone by analysing DRG data. To this end, own empirical surveys are needed. This clear separation was not carried out in most preliminary studies (Resch et al., 2009; Wernitz et al., 2005) and therefore needs further clarification.

\section{Conclusions}

In recent years some improvements have been achieved to illustrate MDRO-hospital infections in the German DRG system. Nevertheless, a deficit between reimbursement and true costs remains, which is still not covered. The hospitals must be unloaded thereof under consideration of their tight financial budgets. It is the task of politics to investigate this problem in more detail and to implement appropriate improvements in near future.

\section{Acknowledgement}

The study is part of the collaborative project "HICARE - Health region Baltic Sea coast, coalition against multiresistant pathogens" funded by the German Ministry of Education and Research (BMBF, Grant agreement number: 01KQ1001C) and the State of Mecklenburg-Western Pomerania (Grant agreement number: UG 11009). We thank all HARMONIC Study colleagues who have contributed to the empirical data collection.

\section{References}

Chin, N., Perera, P., Roberts, A., Nagappan, R. (2013), Review of medical discharge summaries and medical documentation in a metropolitan hospital: impact on diagnosticrelated groups and Weighted Inlier Equivalent Separation, Intern Med J, 43, pp. 767771.

Cosgrove, S. E., Qi, Y., Kaye, K. S., Harbarth, S., Karchmer, A. W., Carmeli, Y. (2005), The impact of methicillin resistance in Staphylococcus aureus bacteremia on patient outcomes: mortality, length of stay, and hospital charges, Infect Control Hosp Epidemiol, 26, pp. 166-174.

de Kraker, M. E., Davey, P. G., Grundmann, H. (2011), Mortality and hospital stay associated with resistant Staphylococcus aureus and Escherichia coli bacteremia: estimating the burden of antibiotic resistance in Europe, PLoS Med 8, e1001104.

de Zeeuw, J., Baberg, H. T. (2009) Current DRG updates - once again the documentation obsession - exemplified by MRSA, Med Klin (Munich) 104, pp. 392-393.

DeWitt, T. G. (1987), How problematic are nosocomial infections in the DRG reimbursement system? Am J Public Health 77, pp. 542-543.

DKG (2007), German Hospital Federation: Documentation sheet for OPS-sodes 8-987, http://www.dkgev.de/media/file/15854.RS037-14_Anlage2-

Dokumentationsbogen_zu_den_OPS-Kodes_aus_8-987.pdf.

ECDC (2012), European Centre for Disease Prevention and Control, Healthcareassociated infections.

Farbman, L., Avni, T., Rubinovitch, B., Leibovici, L., Paul, M. (2013), Cost-benefit of infection control interventions targeting methicillin-resistant Staphylococcus aureus in hospitals: systematic review, Clin Microbiol Infect 19, E582-593. 
Gerlich, M. G., Piegsa, J., Schafer, C., Hubner, N. O., Wilke, F., Reuter, S., Engel, G., Ewert, R., Claus, F., Hubner, C., Ried, W., Flessa, S., Kramer, A., Hoffmann, W. (2015), Improving hospital hygiene to reduce the impact of multidrug-resistant organisms in health care-a prospective controlled multicenter study, BMC Infect Dis 15, p. 441.

Herr, C. E., Heckrodt, T. H., Hofmann, F. A., Schnettler, R., Eikmann, T. F. (2003), Additional costs for preventing the spread of methicillin-resistant Staphylococcus aureus and a strategy for reducing these costs on a surgical ward, Infect Control Hosp Epidemiol 24, pp. 673-678.

Hubner, C., Hubner, N. O., Hopert, K., Maletzki, S., Flessa, S. (2014), Analysis of MRSAattributed costs of hospitalized patients in Germany, Eur J Clin Microbiol Infect Dis 33, pp. 1817-1822.

Jarvis, W. R. (1996), Selected aspects of the socioeconomic impact of nosocomial infections: morbidity, mortality, cost, and prevention, Infect Control Hosp Epidemiol 17, pp. 552557.

Resch, A., Wilke, M., Fink, C. (2009), The cost of resistance: incremental cost of methicillinresistant Staphylococcus aureus (MRSA) in German hospitals, Eur J Health Econ 10, pp. 287-297.

Rosof, B. (2012), The importance of accurate data in quality-of-care measurement, Ann Intern Med 157, pp. 379-380.

Statistika (2014), Average lengths of stay in German hospitals from 1992 to 2014 in days, http:/de.statista.com/statistik/daten/studie/2604/umfrage/durchschnittlicheverweildauer-im-krankenhaus-seit-1992/

Vegni, F. E., Panceri, M. L., Biffi, M., Banfi, E., Porretta, A. D., Privitera, G. (2004), Three scenarios of clinical claim reimbursement for nosocomial infection: the good, the bad, and the ugly, J Hosp Infect 56, pp. 150-155.

Wenzel, R. P. (1995), The Lowbury Lecture. The economics of nosocomial infections, J Hosp Infect 31, pp. 79-87.

Wernitz, M. H., Keck, S., Swidsinski, S., Schulz, S., Veit, S. K. (2005), Cost analysis of a hospital-wide selective screening programme for methicillin-resistant Staphylococcus aureus (MRSA) carriers in the context of diagnosis related groups (DRG) payment, Clin Microbiol Infect 11, pp. 466-471.

Wilcox, M. H. (2004), Health-care-associated infection: morbidity, mortality and costs, Hosp Med 65, pp. 88-91.

Zimlichman, E., Henderson, D., Tamir, O., Franz, C., Song, P., Yamin, C. K., Keohane, C., Denham, C. R., Bates, D. W. (2013), Health care-associated infections: a meta-analysis of costs and financial impact on the US health care system, JAMA Intern Med 173, pp. 2039-2046. 